\title{
Development of a real-time algorithm for detection of the divertor detachment radiation front using multi-spectral imaging
}

\author{
T. Ravensbergen ${ }^{1,2}$, M. van Berkel $^{1}$, S.A. Silburn ${ }^{3}$, J.R. Harrison ${ }^{3}$, A. Perek ${ }^{1}$, K. Verhaegh ${ }^{3}$, W.A.J. Vijvers ${ }^{1 \ddagger}$, \\ C. Theiler ${ }^{4}$, A. Kirk ${ }^{3}$, M.R. de Baar ${ }^{1,2}$, \\ The EUROfusion MST1 team, and the TCV team ${ }^{\dagger}$ \\ April 21, 2020 \\ ${ }^{1}$ DIFFER-Dutch Institute for Fundamental Energy Research, De Zaale 20, 5612 AJ Eindhoven, Netherlands \\ ${ }^{2}$ Eindhoven University of Technology, Department of Mechanical Engineering, Control Systems Technology Group, PO \\ Box 513, 5600 MB Eindhoven, Netherlands \\ ${ }^{3}$ CCFE, Culham Science Centre, Abingdon, Oxon, OX14 3DB, United Kingdom \\ ${ }^{4}$ Ecole Polytechnique Fédérale de Lausanne (EPFL), Swiss Plasma Center (SPC), CH-1015 Lausanne, Switzerland \\ $\ddagger$ Present address: Chromodynamics B.V., High Tech Campus 12, 5656 AE Eindhoven, the Netherlands
}

\begin{abstract}
In this paper we present a novel algorithm to extract the optical plasma boundary and radiation front for detached divertor plasmas. We show that reliable detection of the divertor leg and radiation front is possible using lightweight image processing tools. Using a non-tomographic approach, the detected divertor leg and radiation front can be mapped to the poloidal plane. This approach is fast and accurate enough for real-time control purposes, allowing in particular real-time plasma shape and detachment control, and post-shot detachment physics and dynamics analysis.
\end{abstract}

\section{Introduction}

One of the major challenges in realising a commercially viable fusion reactor is the handling of the power and particle exhaust. In a tokamak fusion device, closed magnetic field lines confining the core plasma are surrounded by open field lines in the scrape-off-layer (SOL) region. Various shaping coils surrounding the vacuum vessel are used to control the magnetic equilibrium. By adding a null ('Xpoint') to the magnetic geometry, better separation of the core plasma from the plasma-wall interaction regions can be achieved, diverting the heat and plasma leaking out of the core to the divertor region. Cross-field transport causes high heat fluxes to enter the SOL, which are transported into this divertor region. Without precautions, the heat fluxes on the divertor targets in next generation fusion devices such as ITER and DEMO will be well above the material limit of $10 \mathrm{MW} / \mathrm{m}^{3}$ [1]. One proposed solution is so-called divertor plasma detachment, which leads to a simultaneous reduction of plasma temperature and ion flux at the divertor target. Detachment is widely studied both experimentally and in terms of modelling, extensively summarized in for example [2] or [3]. Here, we only briefly discuss the aspects relevant for this work. Volumetric radiative dissipation is not sufficient to achieve a reduction (roll-over) in both the ion flux $\Gamma_{i o n}[4]$ and target temperature $T_{t}$. A low plasma pressure $p_{t}$ is necessary, since the ion flux $\Gamma_{i o n}$ is a function of the plasma pressure $\left(\Gamma_{i o n} \propto p_{t} T_{t}^{-1 / 2}\right)$ [5]. To keep the upstream pressure unaffected, necessary for core plasma performance, momentum losses need to be introduced along the SOL to achieve a low $p_{t}$. Detachment is a collection of volumetric atomic and molecular reactions that can lead to significant momentum losses, at the same time introducing significant power and particle losses along the SOL. These atomic and molecular reactions take place

\footnotetext{
*see author list of B. Labit et al. 2019 Nucl. Fusion 59086020

${ }^{\dagger}$ See author list of S. Coda et al. 2019 Nucl. Fusion 59112023
} 
in different temperature and density regimes, and thus, considering the temperature gradient in the divertor, spatially (2D) different parts of the divertor plasma. While being favorable for target erosion and heat flux, the detached regime has been observed to be prone to radiative instabilities [6] or radiative collapses originating from a MARFE instability [7]. The dynamics of detached divertor plasmas are known to differ between existing devices and plasma confinement modes, varying from bifurcation-like jumps of cold regions to the X-point $[6,8]$ to slow progressive evolution on TCV in L-mode [9] and ELM free H-mode [10]. The fronts of these cold regions are often referred to as thermal fronts or detachment fronts. These fronts are considered to be regions of steep temperature gradients, and therefore also represent the front of regions where a particular process in the cold detached divertor plasma dominates. Examples of such processes include charge exchange dominating over ionisation $(\sim 5$ $\mathrm{eV})$, or volumetric recombination $(\sim 1 \mathrm{eV})[11]$. These detachment fronts are observed to move all the way from the target towards the x-point [5], where it can lead to confinement degradation and facilitate easier penetration of neutrals and impurity across the separatrix [12]. Clearly, active real-time feedback control of the detachment front location is a requirement to maintain any divertor solution and prevent target degradation $[5,13]$. Consequently, real-time usage of plasma diagnostics is required. Therefore, in this paper, we present and motivate a novel technique to fully reconstruct the outer divertor leg and emission front location in the poloidal plane using optical boundary reconstruction. This method is real-time capable, such that embedding in (future) feedback control experiments is possible.

The remainder of this paper is organized as follows. After a brief overview on equilibrium reconstruction and detachment control, section 3 introduces our approach and motivation, and the working principle of the detection algorithm is shown. In section 4 the approach to map image coordinates to tokamak coordinates is briefly discussed. Then, in Section 5, the performance of the algorithm is shown, including direct comparison with tomographic inversions and magnetic reconstruction methods.

\section{Background}

\subsection{Boundary reconstruction for plasma shape control}

Magnetic equilibrium reconstruction for control purposes is usually performed using real-time codes like rtEFIT [14] or RT-LIUQE [15]. These codes take flux loop measurements and coil currents as inputs. Additional inputs can be provided by magnetic probes, and sometimes estimates of the plasma current and the diamagnetic flux. However, for prolonged plasma discharges, the inherent integration drift of these measurements and estimates is expected to yield inaccurate equilibrium estimates [16]. Optical boundary reconstruction has been suggested as an alternative or to correct the magnetic reconstructions, as this technique does not suffer from integration drift [17, 18]. Further, optical reconstruction is more reliable when regions of magnetic null are larger [18], for example divertor plasmas with large flux expansion and snowflake configurations. This is especially relevant for alternative divertor solutions [19]. Taking this optical boundary to be the plasma boundary yields sufficiently accurate results for shape control and equilibrium reconstruction purposes $[17,20]$. We will show that optical boundary reconstruction is readily applicable to the detached divertor plasma.

\subsection{Real-time control of divertor detachment}

In previous detachment experiments on the JET tokamak, real-time measurements of $J_{\text {sat }}$ were used for successful heat flux mitigation [21]. Feedback control using divertor tile current measurements is routinely applied on ASDEX Upgrade, which was the first demonstration of succesful divertor heat flux mitigation via feedback [22]. Another successful approach is approximating the inversion of three bolometry channels by taking their weighted sum [23]. On Alcator C-Mod, surface thermocouples were used in real-time to control the tile temperature [24]. DIII-D recently developed a real-time infrastructure to estimate the radiated power in the divertor using 12 bolometry channels [25]. In all these experiments, feedback control with neutral gas injection was applied, using gas valves embedded in the divertor tiles. However, on EAST, the much faster supersonic molecular beam injection (SMBI) system was used in feedback, with additional gas injection programmed as a feedforward waveform 
[26]. During those experiments, the AXUV diagnostic was used to estimate the radiated power in real-time. Finally, on TCV neon seeding experiments have been performed using feedback control, relying on real-time measurements of the intensity of the UV Ne-VIII line at $77 \mathrm{~nm}$, measured by the V-UV diagnostic [27].

\subsection{Motivation}

The experiments listed in Section 2.2 routinely achieved successful lowering of the divertor heat load. However, as detachment is a volumetric phenomenon, having the ability to perform volumetric measurements is a strong advantage in detachment studies and its control. In this work, we therefore propose a novel approach based on real-time analysis of camera images originating from multi-spectral imaging, which provide information on the location of the various emission fronts, and in certain conditions, the impurity radiation front. Controlling the impurity radiation front is important for obtaining simultaneously acceptable core performance (avoiding the movement of the radiation front beyond the x-point) and an acceptably large cold temperature region in the divertor, and to experimentally characterize such trade-off [5]. Additionally, 'conventional' target temperature measurements with Langmuir probes $(<5 \mathrm{eV}[28])$, as well as divertor Thomson scattering $(<1 \mathrm{eV}[29])$, become unreliable in (strongly) detached conditions.

The reconstruction method presented in this work needs to be both accurate and fast enough for its use in real-time feedback control. In this work, the real-time requirement for the imaging system is set by the gas valves used for feedback control. Taking into account their intrinsic delay in opening, gas transport in the divertor, and subsequent response of the plasma, their typical time scale is around $\sim 15 \mathrm{~ms}$. To prevent signal processing issues like aliasing and ghosting, the Nyquist-Shannon theorem [30] states sampling should be at least twice as fast. As a rule of thumb in control engineering practice, a factor $\sim 8-10$ is often used, implying any real-time camera analysis must be performed within $\sim 2$ ms. This is compatible with hardware performance of the MANTIS diagnostic, which typically operates at $200-800 \mathrm{~Hz}[31]$.

The desired level of control performance and post-shot reconstruction determine the required accuracy of optical reconstruction of the divertor leg and emission front. The radial accuracy of the reconstructed plasma edge are extensively discussed in $[17,18]$ and less relevant for detachment control purposes. Here, we focus mainly on reconstruction errors in the distance to the target, which is the relevant parameter for control. In principle, the purpose of real-time detachment control is to prevent excessive heat loads on the divertor tiles while preventing core confinement degradation. However, the targets and performance requirements for detachment control remain to be defined [13], such that requirements on the accuracy of emission front reconstruction are uncertain. Nevertheless, the accuracy of our real-time method compared to conventional post-shot reconstructions is discussed in Sections 4.2 and 5.3.

\subsection{Multi-spectral imaging as divertor diagnostic}

In the TCV tokamak, the C-III emission front at $465 \mathrm{~nm}$ wavelength is found to be a good indicator for the impurity radiation front position [9]. This emission front is defined as the location where a 50 percent emissivity decrease along the divertor leg is first achieved in tomographically inverted images [32, 10]. The unique capabilities of the MANTIS multispectral imaging system [33, 31] allow real-time analysis of spectrally filtered images using lightweight algorithms. In the following, such an algorithm and its analysis capabilities are introduced to approximate the poloidal location of the C-III emission front. Analysis is performed on MANTIS images, and on synthetic C-III images from the MAST-U tokamak using the CHERAB synthetic diagnostic ray-tracing package [34] based on SOLPS simulations of a core density ramp. While TCV has a very open divertor geometry, facilitating spectral imaging, MAST-U has a more complicated divertor structure (super-X compared to the conventional TCV case) and more limited diagnostic access, making it a useful test case for our algorithm. While in this paper attention is given to C-III filtered images, similar emission characteristics are observed in He-II $(468 \mathrm{~nm})$. However, due to its different radiating properties, the He-II emission front can be at a different poloidal location. Further, a possible advantage of multispectral imaging for control is 
the availability of multiple spectrally filtered images simultaneously in real-time. This allows usage of multiple spectral lines in the control loop. However, a physics basis for such a routine is lacking so far, as interpretation and better understanding of spectroscopic data originating from detached plasmas is still being developed [35].

\section{Real-time capable divertor leg and emission front detection}

In this section, our procedure to detect and reconstruct the divertor plasma is introduced. First, we motivate the non-tomographic approach by comparing our analysis with a full tomographic inversion and magnetic reconstruction of the divertor plasma. In Figure 1a) a representative raw C-III filtered input image from MANTIS is shown during the onset of detachment, e.g. just before the roll-over in the target particle flux. The low-field side in this image is on the right. Based on this image, a
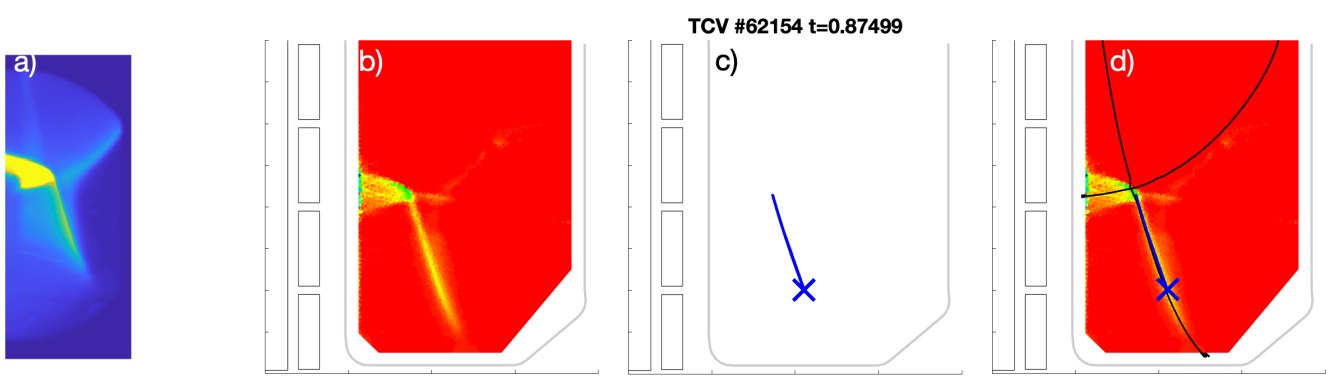

Figure 1: a) Raw C-III filtered image of a detached TCV plasma. Note that the low-field side is located on the right. b) Tomographic inversion of a C-III filtered camera image in the HSV representation. c) Non-tomographic localization of the C-III emission as presented in this paper. d) Both approaches plotted together, with additionally the LIUQE reconstruction of the LCFS in dashed black. Clearly, it can be concluded that, especially for control purposes, the differences (less than $4 \mathrm{~mm}$ for the radial position of the reconstructed divertor legs) are negligible.

post-shot tomographic inversion is performed [10,32] which is shown in Figure 1b). The figure shows a sharp decay of the C-III emission region, indicative of this cold front having left the target. In $c$ ) the novel non-tomographic reconstruction is shown for the same raw input image. Then, in $d$ ) the two approaches are plotted together, along with the LIUQE reconstruction of the last-closed-flux-surface (LCFS) using a black line. From this figure two important conclusions can be drawn. Firstly, the close overlap between magnetic and optical boundary reconstruction that was observed earlier [20] is also present in the divertor region. However, since we use spectrally filtered images, the optical boundary can only be reconstructed in regions where emission of the associated wavelength is present. Secondly, even though the emission front is strictly defined as a localized decrease in emissivity along the divertor leg, we can use the same definition for the intensity in the raw image. However, this approximation breaks down when the emission front is in the x-point and/or not coming from a localized region around the divertor leg. These two observations motivate the approach presented in this paper: a real-time capable reconstruction of the divertor leg and C-III emission front without the need for tomographic inversion, based on matured image processing techniques. In the remainder of this section these processing steps are discussed.

In Figure 2 the necessary algorithmic steps are shown for C-III images of the TCV divertor originating from MANTIS. In the remainder of this section, the step needed to extract the relevant features from the raw images are introduced in more detail.

1. First, a fixed, unchanging region-of-interest (ROI) is sub-sampled from the higher resolution MANTIS image, see Figure 2a). This ROI can be rectangular or a circle segment, in which case interpolation of pixel intensities is performed to obtain a rectangular matrix for further processing. This mapping is visualized with colored corners indicating how the semi-circle ROI in the image frame in Figure 2a) is stretched to a square matrix (Figure 2b)). The size of 

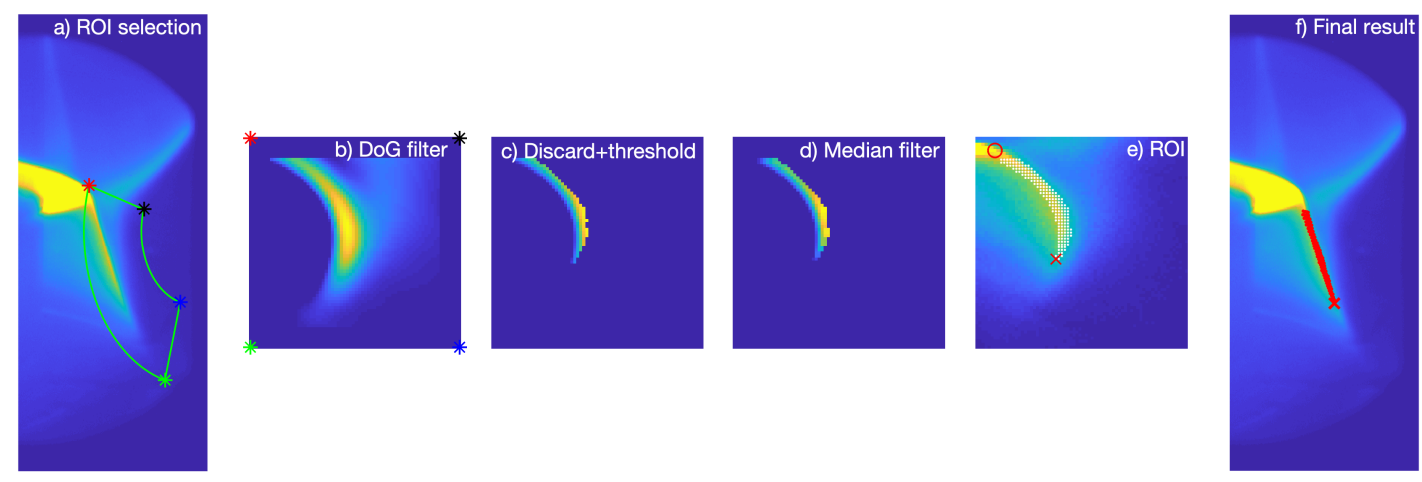

Figure 2: Algorithmic steps to detect the optical divertor boundary and associated emission front, applied to C-III images originating from the MANTIS diagnostic on TCV. a) Sampling of a fixed region-of-interest (ROI), corners indicated with colored asterisks. Linear interpolation of pixel intensities is applied to get square matrices for further processing. b) Convolution with Derivative-of-Gauss (DoG) kernel with original ROI corners indicated. c) Threshold $t_{\epsilon}$ on original image intensity while discarding negative DoG gradients. d) Nonlinear eroding with median filter. e) Final detected divertor leg points in ROI image with location of the maximum intensity (red circle) and emission front (red star). f) Final result in the original image with the divertor leg and front being robustly detected.

the ROI matrix is typically less than $100 \times 100$ pixels, making the consecutive processing steps computationally inexpensive.

2. Next, the convolution of the ROI with a 2D derivative-of-Gauss kernel (DoG) [30] is computed. By sliding over the image, this operation smooths the image, and takes the derivative of its pixel intensity in the horizontal direction. Sharp gradients in pixel intensity arise perpendicular to the divertor leg, such that this operation allows straightforward isolation of the associated divertor leg pixels.The kernel has its derivative in the $x$ - or horizontal pixel direction:

$$
\begin{aligned}
\frac{\partial G}{\partial x} & =-\frac{1}{A} \frac{x}{\sigma^{2}} G(x, y, \sigma), \text { with } \\
G(x, y, \sigma) & =\exp \left(-\frac{x^{2}+y^{2}}{2 \sigma^{2}}\right)
\end{aligned}
$$

In here $G(x, y, \sigma)$ is a Gaussian kernel in the pixel coordinates $(x, y)$, having standard deviation $\sigma$. The constant $A$ is chosen such that the 2 -norm of the DoG-filter is 1 . Note that in practice, the filter has a finite support, here taken to be 15 pixels. By changing $\sigma$ the amount of smoothing can be varied. For our application we choose $\sigma=6$, for which the result is shown in Figure $2 b)$. Computing the convolution between ROI-matrix $R(k, l)$ (indexing via column-major matrix coordinates) and DoG-matrix $G_{x}$ convolution equation results in a matrix $C$ with elements $(i, j)$ (again in column-major matrix coordinates):

$$
C(i, j)=\sum_{k} \sum_{l} R(k, l) G_{x}(i-k+1, j-l+1)
$$

Note that $k$ and $l$ run only over values that result in legal indices for both $R$ and $G_{x}$. Boundary pixels in the ROI therefore require (zero-)padding to compute $C$. Here, we only compute the convolution in the 'valid' region to prevent fictitious gradients due to zero-padding around the ROI.

3. After the convolution with the DoG, negative gradients are discarded, and a threshold $t_{\epsilon}$ is applied on the convoluted image using the pixel intensity in the original ROI. By carefully tuning this threshold $t_{\epsilon}$, the selected region in the DoG-filtered image is bounded by the emission front, as points further downstream have a lower intensity. The remaining pixels have a positive intensity gradient and at least intensity $t_{\epsilon}$ in the original frame. Together, these two properties bound the divertor leg and its emission front. This is shown in Figure 2c). 
4. Consecutive nonlinear median filtering with support $N=3$ greatly reduces residual salt-andpepper noise [36], cf. Figure 2d).

5. The pixels that are left after median filtering comprise the divertor leg (characterized by a sharp gradient), and are bounded by the emission front (due to the $t_{\epsilon}$ threshold). Any outlier pixels are eroded by the median filter. The residual pixels are shown in the ROI frame in Figure 2e) as white dots. The maximum used for the threshold $t_{\epsilon}$ is indicated by a red circle, and the location of the emission front is marked with a red $X$. Finally, the residual pixels are transformed back to the original image, and the emission front is again marked with a red $X$, see Figure $2 f$ ).

Next, the detected divertor leg and emission front need to be mapped to the poloidal plane, which is discussed in the following section.

\section{Coordinate transform: Mapping to poloidal plane}

A common method to map pixel coordinates in $\mathbb{R}^{2}$ to a world frame in $\mathbb{R}^{3}$ is to retrace a suitable modelled camera projection. The classic pinhole camera model [37] can be used to project a set of world coordinates $(X, Y, Z) \in \mathbb{R}^{3}$ expressed in the Cartesian world frame (denoted by $w$ ) onto pixel coordinates $(x, y)$. The camera is located on position $\vec{x}_{c a m}^{w}$ expressed in the world frame. The camera has its own local frame indicated by $c$, which has its $z$-axis pointing along its optical axis. Then, the camera projection can be described by:

$$
\left(\begin{array}{l}
x \\
y \\
1
\end{array}\right)=K\left(\begin{array}{ll}
R^{c w} & -R^{c w} \vec{x}_{c a m}^{w}
\end{array}\right)\left(\begin{array}{c}
X \\
Y \\
Z
\end{array}\right) .
$$

In here, the (by definition) orthogonal rotation matrix $R^{c w} \in \mathbb{R}^{3 \times 3}$ rotates the world frame $w$ to the camera frame $c$, and matrix $K \in \mathbb{R}^{3 \times 3}$ contains the intrinsic camera parameters. These intrinsic and extrinsic camera properties can be obtained by camera calibration, here performed using the CALCAM routine [38]. This routine also takes radial and tangential lens distortion into account, by fitting a nonlinear distortion correction model, known as the Brown-Conrady model [39]:

$$
\left(\begin{array}{l}
x_{n} \\
y_{n}
\end{array}\right)=\left(1+k_{1} r^{2}+k_{2} r^{4}\right)\left(\begin{array}{l}
x_{d} \\
y_{d}
\end{array}\right)+\left(\begin{array}{l}
2 p_{1} x_{d} y_{d}+p_{2}\left(r^{2}+2 x_{d}^{2}\right) \\
p_{1}\left(r^{2}+2 y_{d}^{2}\right)+2 p_{2} x_{d} y_{d}
\end{array}\right)
$$

Here, subscript $n$ denotes the normalized undistorted pixel coordinates, and $d$ the distorted coordinates. The radius $r$ is defined as $r \equiv \sqrt{x_{d}^{2}+y_{d}^{2}}$. Note that for real-time undistortion of pixel coordinates, we apply a distortion correction model of the form $x_{n}=f\left(x_{d}\right)$, not the distortion model $x_{d}=g\left(x_{n}\right)$ implemented by popular packages like openCV [40]. The latter requires solving a nonlinear equation for every distorted pixel, which is not applicable to our real-time application.

Clearly, the inverse mapping from pixel to world coordinates is not unique, as each measured pixel intensity is the result of integrated emission along the sight line. This sight line, however, can be uniquely determined. For physics analysis, computationally demanding tomographic inversions are then applied to determine emissivity plots [9]. Here, we apply a faster non-tomographic approach presented in [41]. In this approach, the sight lines associated with the divertor leg and emission front are projected onto the vertical plane $X=0$ passing through the tokamak center. All the residual points comprising the divertor leg are mapped onto this plane to determine their coordinates $\left(Y_{0}, Z_{0}\right)$. Then, a second order polynomial is fitted through these coordinates. The order of the polynomial is sufficiently high to capture the relevant shape, but is also low enough to have a desired analytical property, which is explained later in section 4.1. From the camera location in the world frame and the coordinates $\left(Y_{0}, Z_{0}\right)$ in the plane $X=0$, the point of tangency $(R, Z)$ between the camera sightline and the plasma can be uniquely determined, cf. [41, Fig. 4]. This location can be uniquely recovered when axisymmetry of the plasma is assumed and a (polynomial) fit with analytical derivative is available. More details on the mapping method are provided in [41]. 
In Section 3, it has already been discussed that for the cases of interest here, even though the image is not tomographically inverted, the resulting emission front location is close to the one obtained from full tomographic inversion, cf. Figure 1. In section 4.2 the consistency and error of the reconstruction is discussed, and in Section 5.3 a qualitative comparison between the full tomographic inversion and our real-time approximation is performed. The quantity that is compared is the distance along the divertor leg to the target, which is introduced next.

\subsection{Calculation of the distance along the divertor leg to the target}

The distance along the divertor leg to the divertor target is a useful controllable quantity for future feedback experiments. This quantity is always monotonically increasing when detachment proceeds, which is especially useful for curved divertor leg geometries near the target like in the super-X configuration. Here, we assume that the discharge equilibrium is constant during the time the reconstruction algorithm is actively used, allowing a constant fit of the divertor leg, for example $R_{l e g} \approx p_{2}\left(Z_{l e g}\right)$, with $p_{2}$ a second order polynomial. Then, the length along the LCFS is given by:

$$
L_{\text {pol }}=\int_{Z_{\text {target }}}^{Z_{\text {det }}} \sqrt{1+\left(\frac{\mathrm{d} R_{\text {leg }}}{\mathrm{d} Z_{\text {leg }}}\right)^{2}} \mathrm{~d} Z_{\text {leg }} \approx \int_{Z_{\text {target }}}^{Z_{\text {det }}} \sqrt{1+\left(\frac{\mathrm{d} p_{2}}{\mathrm{~d} Z_{\text {leg }}}\right)^{2}} \mathrm{~d} Z_{\text {leg }} .
$$

In here, $Z_{\text {target }}$ and $Z_{\text {det }}$ are the vertical target position and detected emission front position respectively. For second order polynomials (6) has an analytical solution, making real-time evaluation slightly faster than numerical evaluation of the integral.

\subsection{Emission front reconstruction error}

The error in estimating the emission front location is an accumulation of 1) the approximation in not performing a full tomographic inversion, 2) systematic errors in the image processing steps, and 3 ) a systematic error in the mapping to the poloidal plane. The total error in $L_{p o l}$ between the real-time approximation and the full tomographic analysis is typically less than $4 \mathrm{~cm}$ RMS, which is discussed in more detail in Section 5.3. In this section, we focus on the contribution of the image processing and mapping error. As discussed in section 3, the image processing smooths the image and takes the gradient with a derivative-of-Gauss filter, having a standard deviation of 6 pixels. By definition, the smoothing diffuses the edge feature in the original image in the direction of smoothing. Therefore, in the worst case, the smoothing results in a deviation of 6 pixels of the true edge location. The subsequent mapping error is determined by the accuracy of the Calcam calibration. As the CHERAB forward modeling code uses the same camera model as Calcam, making an error estimate somewhat arbitrary, we continue our error analysis for the MANTIS specific case. Calcam calibrations for MANTIS typically have an root-mean-square deviation of less than 2 pixels. As such, if we take an upper bound of 10 pixels error in image processing, and perform the subsequent mapping to the poloidal plane, the final error in the distance to the target is in the order of $1 \mathrm{~cm}$. This is within the accuracy required for control purposes.

\section{Results}

In this section, we process C-III filtered images originating from two sources: CHERAB synthetic data for the MAST-U tokamak, and real camera data from the MANTIS diagnostic on TCV. For experimental data on TCV, the influence of the target radius on the reconstruction is investigated in Section 5.2, and a direct comparison between further analysis of a full tomographic inversion is made in Section 5.3. This analysis relies on magnetic reconstruction of the divertor leg location, such that it is different to the quantitative comparison already shown in Figure 1.

\subsection{Comparison to magnetic reconstructions}

First, we analyze synthetic data originating from the CHERAB forward modelling code. Given the emission front detection scheme in Figure 2, the resulting detection is shown on the left in Figure 3. 
The ROI is plotted as a green circle segment, the detected divertor leg is shown in red, and the emission front is marked with a red X. The subsequent mapping to the poloidal plane is shown on the right, including a cross section of the MAST-U divertor chamber and surrounding coils. For this simulation case without camera distortion, the reconstruction is near perfect as long as the emission front is not reattaching. During reattachment, the divertor leg becomes horizontal in the super-X configuration, causing tangent sight lines to the plasma to disappear. Next, with the same thresholds and settings in

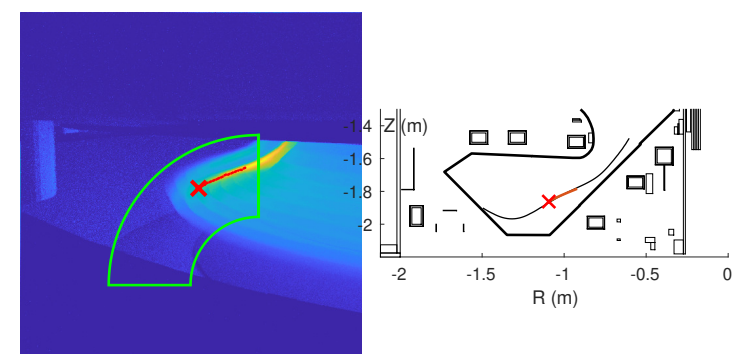

Figure 3: Left: Raw C-III filtered input image from CHERAB for the MAST-U divertor, together with the ROI, the detected divertor leg, and the emission front. Right: Poloidal mapping of the detected divertor leg (red) and emission front (red circle) in a cross-section of the MAST-U divertor chamber, together with the LCFS in black. The divertor coils and vacuum vessel are also plotted.

the image processing algorithm as for MAST-U, the analysis is performed on images of a representative detached single-null TCV plasma, originating from the MANTIS system. The resulting detection is shown on the left in Figure 4. In the same plot on the right, the magnetic reconstruction of the LCFS from LIUQE is shown in black. The difference between the magnetic reconstruction of the LCFS and the optical boundary is less than $4 \mathrm{~mm}$ for the radial position of both reconstructed divertor legs: Even in this case of finite non-zero camera distortion for a real experiment. A typical analysis as

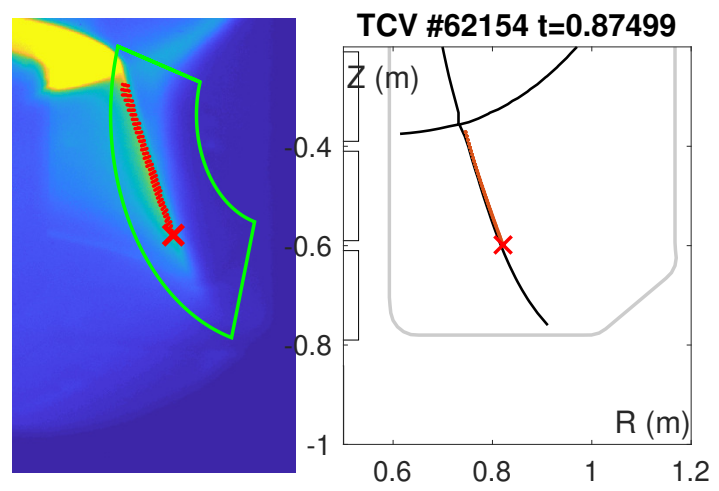

Figure 4: Left: Original image originating from MANTIS, with ROI indicated. Note again the image orientation with the LFS on the right-hand side. Poloidal mapping of the optical boundary of TCV discharge \#62154 at $t=0.87 \mathrm{~s}$, together with the LIUQE reconstruction of the LCFS. Clearly, the difference is marginal, being less than $4 \mathrm{~mm}$ in terms of the radial position of the reconstructed divertor legs.

shown here takes on average $1 \mathrm{~ms}$ per frame on a regular 2019 dual core laptop processor, utilizing non-optimized, compiled Matlab Simulink code ${ }^{1}$. As MANTIS operates at least on $400 \mathrm{~Hz}$ sampling rate, the analysis is fast enough to meet current acquisition speeds. If necessary, further speedup can be obtained utilizing a dedicated computational node in the plasma control system, having faster and newer hardware and optimized compiled code. Nevertheless, with the current processing time, the real-time control requirement set in Section 2.2 is met.

\footnotetext{
${ }^{1}$ https://www . mathworks. com/products/simulink.html
} 


\subsection{Detection for varying the target radius}

To verify whether the target radius has influence on the accuracy of the optical reconstruction of the divertor leg, we compare three TCV discharges with varying target radii. Discharges \#62152 $\left(R_{t}=0.68 \mathrm{~m}\right), \# 62135\left(R_{t}=0.75 \mathrm{~m}\right)$, and \#62154 $\left(R_{t}=0.90 \mathrm{~m}\right)$ are otherwise similar, all having a poloidal flux expansion of $\sim 5$. In Figure 5 the magnetic equilibria are plotted together with the optical reconstructions for the three discharges under consideration. Clearly, for all considered cases,

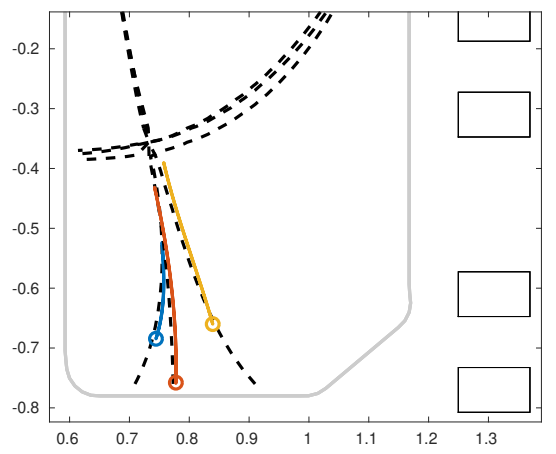

Figure 5: Magnetic equilibria (dashed black) and optical reconstruction for three different target radii: TCV \#62152 $\left(R_{t}=0.68 \mathrm{~m}\right)$ in blue, \#62135 $\left(R_{t}=0.75 \mathrm{~m}\right)$ in red, and \#62154 $\left(R_{t}=0.90 \mathrm{~m}\right)$ in yellow. Divertor leg reconstruction quality is similar for all considered cases.

the reconstruction is sufficiently accurate for our control purposes.

\subsection{Comparison with analysis on full tomographic inversions}

In section 3 it was already discussed that the approximation of taking a line integrated image to reconstruct the emission front breaks down when the (actual) emission front comes close to the x-point. In this section, we directly compare the real-time reconstruction of the emission front based on raw, lineintegrated images, with analysis on full tomographic inversions. A common approach in such analysis [9] is to take the divertor leg location in the poloidal plane from magnetic equilibrium reconstruction, and determine the 50 percent emissivity decrease in a group of pixels of the tomographically inverted image surrounding the magnetically reconstructed divertor leg. Clearly, this leads to a combination of optical methods with magnetic reconstruction, such that a comparison with our real-time approach is not a direct comparison between two optical methods. It was already discussed in Section 2.1 that magnetic reconstruction can deviate significantly in some configurations. However, taking a group of pixels around the magnetically reconstructed divertor leg allows for some deviation between the magnetic reconstruction and tomographic inversion. Nevertheless, for our comparison, we choose a discharge where the optical boundary in the tomographic inversion are close. An example of such a discharge is TCV \#65349, which is a detached single-null L-mode experiment with $I_{p}=320 \mathrm{kA}$ and significant movement of the C-III front. First, in Figure 6 we plot the tomographic inversion in the divertor region at $t=0.95 \mathrm{~s}$ together with both reconstruction methods and the magnetic boundary. The LCFS is plotted in dark dashed blue, with in dashed cyan the bounds of the poloidal group of pixels that was used to allow some error between LIUQE and the tomographic inversion. The detected divertor leg and emission front as reconstructed with the real-time algorithm presented in this paper is plotted in black, with the emission front marked as a black cross. Furthermore, some artifacts of the inversion can be observed in the top right corner, which are not relevant for this analysis. Like in Section 3, the correspondence between both optical methods (tomographic and real-time non-tomographic) with the magnetic reconstruction is apparent. There is a small error between the tomographically reconstructed location of the emission front and our real-time reconstruction. However, if we plot $L_{p o l}$ for both methods for the whole discharge under consideration in Figure 7 , we see that the particular time frame of Figure $6(t=0.95 \mathrm{~s})$ is among the worst cases. The approximation error stays below $6 \mathrm{~cm}$ for the whole duration of the discharge, with an RMS error of $4 \mathrm{~cm}$. Further, we see the two methods are almost identical for front positions around the middle and lower end of 
the divertor leg. The error between the two signals is separately plotted in Figure 8. As the poloidal leg length for this particular plasma scenario is around $40 \mathrm{~cm}$, the relative error is below $10 \%$.

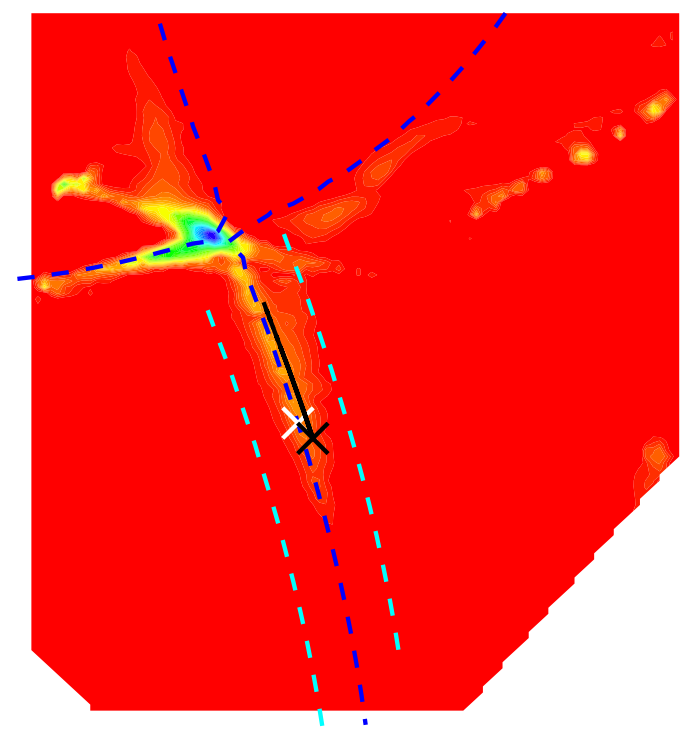

Figure 6: Tomographic inversion at $t=0.95 \mathrm{~s}$ in the divertor region together with magnetic reconstruction of the LCFS and divertor leg (blue dashed line). The tube region which is used to find the C-III front location for the mixed tomographic/magnetic reconstruction method is plotted in dashed cyan. The corresponding emission front is indicted with a white cross. The detected divertor leg using the real-time approach is plotted in black, with the emission front marked with a black cross. The mismatch in both emission front locations is small, but in fact among the largest for the discharge under consideration: See Figure 7.

\section{Discussion and outlook}

In this paper, we apply matured image processing tools to experimental and synthetic multi-spectrally filtered camera data to reconstruct the divertor plasma boundary and emission front in detached tokamak plasmas. Using a non-tomographic inversion procedure, a poloidal reconstruction of the divertor plasma can be obtained, which closely matches the magnetic reconstruction of the divertor leg from established reconstruction codes. The non-tomographic approach is compared to a full tomographic procedure. The results are plotted in the poloidal plane and it is shown that the correspondence of the two methods is good. Besides this direct quantitative comparison between two optical methods, a third method is qualitatively compared to the real-time capable reconstruction, which relies on magnetic reconstruction methods to find the divertor leg. The error between this mixed method and the real-time reconstruction is around $4 \mathrm{~cm}$ root-mean-square in the poloidal plane, with the largest systematic error for front positions close to the X-point. The real-time reconstruction approach presented in this paper is therefore promising for application in dynamic characterization of detachment dynamics, called system identification, in which perturbative experiments are carried out to analyze the movement of the emission front as a function of the applied neutral gas puffing in a formalized and systematic fashion. From these experiments data-driven models, or transfer functions, can be extracted which can be directly used for offline controller design [42]. The results shown in this paper have been obtained using post-processing of our real-time capable algorithms. Recent results on new experiments have however shown identical performance during real-time feedback experiments [43].

For application in next generation devices, the radiating species are different, and the control loop should be based on intrinsic plasma species such as hydrogen and He-II. For example, in [44] the hydrogenic radiation front is analysed using high-n Balmer series. These emissions can be used to directly derive characteristics on the atomic and/or molecular reactions driving the detachment dynamics, such as ionisation and recombination [45]. With the techniques developed in this paper, together with the MANTIS diagnostic and further developments in spectral interpretation, one could work towards a control loop based on the monitoring of such processes directly. Furthermore, the 


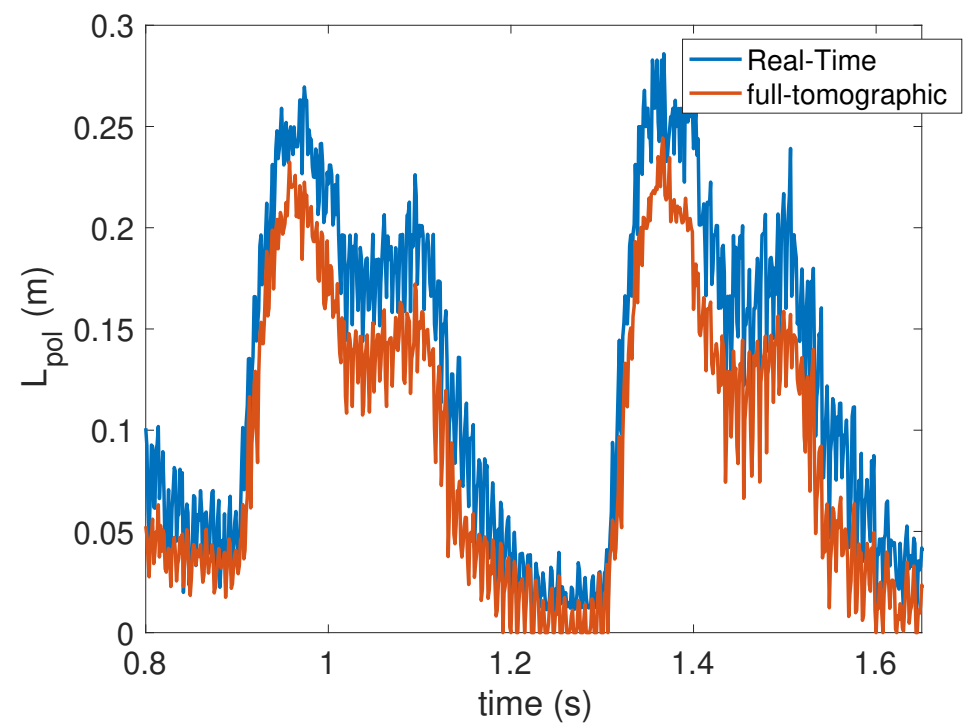

Figure 7: Comparison between the C-III front tracking for the tomographic/magnetic approach (orange) and the real-time analysis presented in this paper (blue). As discussed in Section 3, the reconstruction error becomes larger for front locations closer to the x-point. However, the overall error is below $6 \mathrm{~cm}$, with $<4 \mathrm{~cm}$ RMS which is sufficient for control.

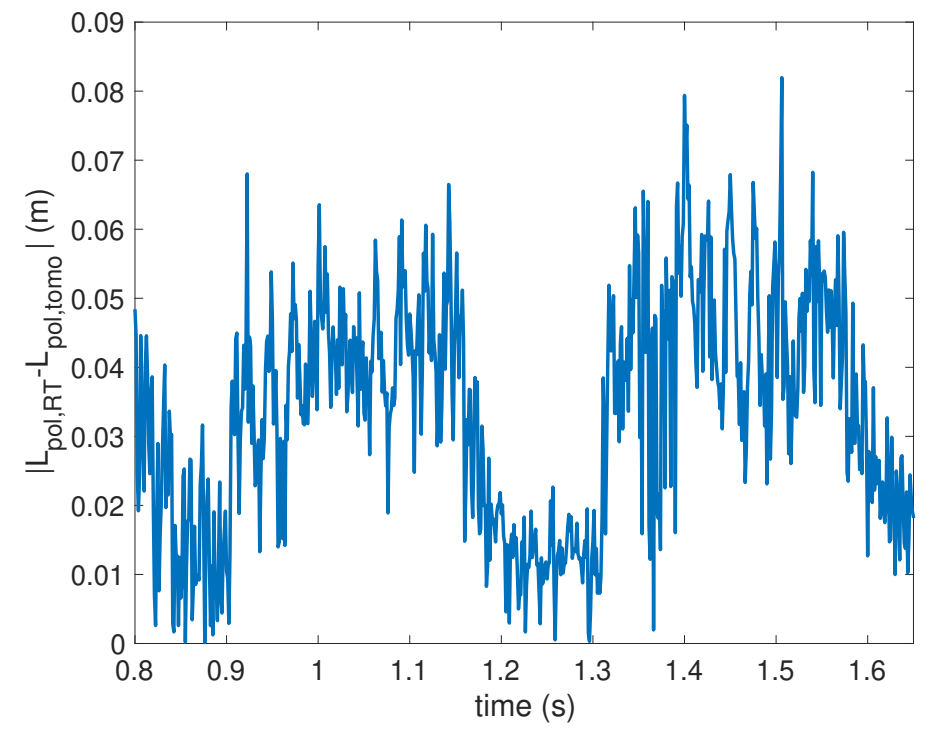

Figure 8: Error in terms of $L_{p o l}$ between the analysis of tomographically inverted images and the real-time algorithm discussed in this paper. The error stays below $6 \mathrm{~cm}$ with an RMS error of $4 \mathrm{~cm}$, which is accurate enough for control purposes and quick post-shot analysis. 
2D coverage and reconstruction approach allows for inclusion of, for example, strike-point sweeping control on the divertor target. For such an application, the ROI has to be modified to include the whole swept volume, or must be updated during the discharge.

It was already discussed that the existing MANTIS hardware is ready for the real-time interpretation of multiple spectral lines [31]. However, the real-time capability is not only set by the time scales concerning the gas fuelling, but more importantly the dynamics of the detachment behaviour. These dynamics are strongly device dependent and can change between $\mathrm{H}$ - and L-mode. In TCV, a gradual evolution of detachment with further gas fuelling is observed and, consequentially, the C-III emission region is observed to move gradually towards the $\mathrm{x}$-point. On other devices, however, a detachment 'cliff' [8] can be observed where the divertor goes into a bifurcation-like state where small changes in fuelling result in jumps in $T_{e}$ from $\sim 10 \mathrm{eV}$ to $\sim 1 \mathrm{eV}[8]$; likely resulting in a higher sensitivity of the C-III emission front to gas fuelling. There are several divertor parameters which can influence this sensitivity, such as the magnetic field profile along the divertor leg $[5,9]$. It also, likely, depends on the precise balance between momentum and power losses [46].

The quality of the emission front reconstruction could be characterized by the local intensity gradient around the front, which could further improve the reliability of the algorithm. Unfortunately, cameras are known to quickly break down in a nuclear environment. For application in ITER and/or DEMO, shielding options and mirror cleaning techniques should be investigated. Also, as opposed to carbon, the wall material is highly reflective, possibly lowering the quality of the reconstruction.Fortunately, the CHERAB code allows for calculation of reflection for the chosen camera field-of-view, which can be done offline [47]. Then the reflections can be accounted for in the subsequent real-time analysis. Further reliability of the detection can be obtained by combining it with other diagnostics, e.g. bolometry and AXUV. In control engineering practice, such sensor fusion is often realized in real-time using a Kalman filter approach [42], which relies on fast physics-based models that are evaluated along with the discharge. The development of such models is aided by the aforementioned system identification, as both model and experiment lead to a transfer function characterization of the dynamic behavior which can be directly compared.

\section{Acknowledgments}

The first author would like to thank valuable input from the TCV team, especially M. Wensing. The TCV LIUQE team is kindly acknowledged for their input on the comparison of reconstruction methods. Also fruitful discussions with Dr. Bernert from IPP Garching are greatly acknowledged. Dr. Moulton from UKAEA is kindly acknowledged for providing the SOLPS simulations on which the synthetic MAST-U images are based. This work was supported in part by the Swiss National Science Foundation. This work has been carried out within the framework of the EUROfusion Consortium and has received funding from the Euratom research and training programme 2014-2018 and 2019-2020 under grant agreement No 633053. The views and opinions expressed herein do not necessarily reflect those of the European Commission.

\section{References}

[1] Loarte, A. et al. 2007 Nuclear Fusion 47 S203

[2] Krasheninnikov, S.I. et al. 2017 Journal of Plasma Physics 83155830501

[3] Leonard, A.W. 2018 Plasma Physics and Controlled Fusion 60044001

[4] Loarte, A. et al. 1998 Nuclear Fusion 38331

[5] Lipschultz, B. et al. 2016 Nuclear Fusion 56056007

[6] Reimold, F. et al. 2015 Nuclear Fusion 55033004

[7] Lipschultz, B. 1987 Journal of Nuclear Materials 145-147 15

[8] Jaervinen, A.E. et al. 2018 Physical Review Letters 12175001

[9] Theiler, C. et al. 2017 Nuclear Fusion 57072008

[10] Harrison, J.R. et al. 2019 Plasma Physics and Controlled Fusion 61065024 
[11] Post, D.E. 1995 Journal of Nuclear Materials 220-222 143

[12] Goetz, J.A. et al. 1999 Physics of Plasmas 61899

[13] ITER Organisation 2018 ITER Research Plan within the Staged Approach (Level III - Provisional Version) Technical Report September 2018

[14] Lao, L.L. et al. 1985 Nuclear Fusion 251611

[15] Hofmann, F. et al. 1988 Nuclear Fusion 281871

[16] Hutchinson, I.H. 2002 Principles of Plasma Diagnostics Cambridge University Press second edition

[17] Zhang, H. et al. 2018 IEEE Transactions on Plasma Science 462162

[18] Hommen, G. et al. 2014 Nuclear Fusion 54073018

[19] Reimerdes, H. et al. 2017 Nuclear Fusion 57126007

[20] Hommen, G. 2014 Optical boundary reconstruction for shape control of tokamak plasmas Ph.D. thesis Eindhoven University of Technology

[21] Guillemaut, C. et al. 2017 Plasma Physics and Controlled Fusion 59045001

[22] Kallenbach, A. et al. 2010 Plasma Physics and Controlled Fusion 52055002

[23] Kallenbach, A. et al. 2015 Nuclear Fusion 55053026

[24] Brunner, D. et al. 2016 Review of Scientific Instruments 87023504

[25] Eldon, D. et al. 2019 Nuclear Materials and Energy 18285

[26] Wu, K. et al. 2018 Fusion Engineering and Design 129291

[27] Pitts, R.A. et al. 1999 Journal of Nuclear Materials 266648

[28] Février, O. et al. 2018 Review of Scientific Instruments 89053502

[29] Hawke, J. et al. 2013 Journal of Instrumentation 8 C11010

[30] Mallat, S. 2008 A wavelet tour of signal processing: the sparse way Academic Press third edition ISBN 13:978-0-12-374370-1

[31] Perek, A. et al. 2019 The Review of scientific instruments 90123514

[32] Carr, M. et al. 2018 Review of Scientific Instruments 89083506

[33] Vijvers, W.A.J. et al. 2017 Journal of instrumentation 12 C12058

[34] Carr, M. et al. 2017 in 44th EPS Conference on Plasma Physics Belfast, Northern Ireland O. 5.130

[35] Verhaegh, K. et al. 2017 Nuclear Materials and Energy 121112

[36] Lim, J.S. 1990 Two-Dimensional Signal and Image Processing Prentice-Hall, Englewood Cliffs, New Jersey

[37] Hartley, R. et al. 2003 Multiple View Geometry in Computer Vision Cambridge University Press second edi edition ISBN 9788578110796

[38] Silburn, S. et al. 2018 Calcam, Python tools for spatial calibration of camera diagnostics

[39] Brown, D.C. 1966 Photogrammetric Engineering and Remote Sensing 17444

[40] Bradski, G. et al. 2008 Learning OpenCV: Computer vision with the OpenCV library O'Reilly Media, Inc., Sebastopol, CA, USA first edition ISBN 978-0-596-51613-0

[41] Hommen, G. et al. 2010 Review of Scientific Instruments 81113504

[42] Skogestad, S. et al. 2005 Multivariable Feedback Control Wiley second edition

[43] Ravensbergen, T. et al. 2019 in 46th EPS Conference on Plasma Physics Milan, Italy O3.305

[44] Verhaegh, K. 2018 Spectroscopic investigations of detachment on TCV Ph.D. thesis University of York

[45] Verhaegh, K. et al. 2019 Plasma Physics and Controlled Fusion 61125018

[46] Dudson, B.D. et al. 2019 Plasma Physics and Controlled Fusion 61065008

[47] Carr, M. et al. 2019 Review of Scientific Instruments 90043504 